01

\title{
Фотонные кристаллы на основе сред с произвольной анизотропией диэлектрической и магнитной проницаемостей
}

\author{
(C) Г.Ф. Глинский
}

Санкт-Петербургский государственный электротехнический университет „ЛЭТИ“, 197376 Санкт-Петербург, Россия

e-mail: genaglinskii@mail.ru

(Поступило в Редакцию 14 июля 2018 г.)

\begin{abstract}
Предложен общий подход к анализу собственных мод в анизотропных и гиротропных $3 D$ фотонных кристаллах на основе диэлектрических и магнитных сред. В основе предлагаемого подхода лежит представление стационарных макроскопических уравнений Максвелла в операторной форме, соответствующей квантово-механическому уравнению для фотона, спин которого $s=1$. Напряженностям электрического и магнитного полей в этих уравнениях сопоставлены векторы состояний в комплексном гильбертовом пространстве. Диэлектрическая и магнитная проницаемости выступают в роли операторов, действующих на эти векторы. Показано, что задача определения собственных мод фотонного кристалла сводится к поиску собственных векторов и собственных чисел эрмитова оператора, характеризующего спин-орбитальное взаимодействие фотона в исследуемой периодической анизотропной среде. В качестве базиса для представления операторных уравнений предлагается использовать состояния фотонов с определенным волновым вектором (определенным импульсом) и с определенной линейной или круговой спиновой поляризацией. В качестве примера рассмотрены одномерные фотонные кристаллы. Исследовано влияние анизотропии и гиротропии на дисперсию собственных мод в этих кристаллах. Проанализированы групповая скорость собственных мод, переносимый ими импульс, а также спиновый момент импульса в случае гиротропных сред.
\end{abstract}

DOI: $10.21883 / J T F .2019 .03 .47163 .273-18$

\section{Введение}

Фотонные кристаллы (ФК) как новый вид оптических сред появились благодаря пионерским работам Э. Яблоновича и С. Джона $[1,2]$. В настоящее время на их основе создан ряд новых оптических приборов и устройств таких как оптические фильтры, микрорезонаторы, волноводы, разветвители и др. В большинстве работ, посвященных теоретическому исследованию ФК, авторы, как правило, ограничивались анализом немагнитных сред с периодически изменяющейся в пространстве изотропной диэлектрической проницаемостью [3-6]. Однако одним из наиболее перспективных направлений в области применения ФК является разработка приборов с управляемыми характеристиками. Возможность управления модовой структурой ФК посредством внешних воздействий, например, внешнего электрического или магнитного поля, а также механической деформации, открывает широкие возможности их использования в различных оптоэлектронных устройствах в том числе в параметрических усилителях и преобразователях света. Любые внешние воздействия, приводящие к перестройке собственных мод ФК, так или иначе, связаны с изменением тензора диэлектрической или магнитной проницаемости среды. В частности, индуцируемая внешними полями анизотропия или гиротропия должны приводить к снятию поляризационного вырождения собственных мод изотропного ФК. В настоящее время в литературе отсутствует общий подход к анализу ФК на основе периодических сред с произвольной анизотропией диэлектрической и магнитной проницаемостей. Отметим лишь работы [7-14], в которых исследовались частные случаи анизотропных и гиротропных ФК.

В настоящей работе теория фотонных кристаллов обобщается на случай периодических диэлектрических и магнитных сред с произвольной анизотропией диэлектрической и магнитной проницаемостей, а также сред, обладающих гиротропными свойствами. Предлагается простая операторная форма записи стационарных уравнений Максвелла, аналогичная квантово-механическому уравнению для фотона, спин которого $s=1$. Показано, что задача определения собственных частот и собственных мод ФК сводится к расчету собственных однофотонных состояний, представляющих собой векторы в комплексном гильбертовом пространстве, а также собственных чисел эрмитова оператора, характеризующего спин-орбитальное взаимодействие фотона в исследуемой среде.

\section{1. Операторная форма макроскопических уравнений Максвелла}

Как известно, для описания электромагнитных волн в рамках макроскопической электродинамики в отсутствии источников поля достаточно рассмотреть следующие уравнения Максвелла:

$$
\begin{aligned}
& \nabla \times \mathbf{H}(\mathbf{x}, t)=\frac{1}{c} \frac{\partial}{\partial t} \mathbf{D}(\mathbf{x}, t), \\
& \nabla \times \mathbf{E}(\mathbf{x}, t)=-\frac{1}{c} \frac{\partial}{\partial t} \mathbf{B}(\mathbf{x}, t) .
\end{aligned}
$$


Стационарные, т.е. установившиеся и периодически изменяющиеся во времени решения этих уравнений, с учетом вещественности поля всегда можно представить в виде суммы двух независимых решений

$$
\begin{aligned}
& \mathbf{E}(\mathbf{x}, t)=A\left[\mathbf{E}(\mathbf{x}) e^{-i \omega t}+\mathbf{E}^{*}(\mathbf{x}) e^{i \omega t}\right], \\
& \mathbf{H}(\mathbf{x}, t)=A\left[\mathbf{H}(\mathbf{x}) e^{-i \omega t}+\mathbf{H}^{*}(\mathbf{x}) e^{i \omega t}\right], \\
& \mathbf{D}(\mathbf{x}, t)=A\left[\mathbf{D}(\mathbf{x}) e^{-i \omega t}+\mathbf{D}^{*}(\mathbf{x}) e^{i \omega t}\right], \\
& \mathbf{B}(\mathbf{x}, t)=A\left[\mathbf{B}(\mathbf{x}) e^{-i \omega t}+\mathbf{B}^{*}(\mathbf{x}) e^{i \omega t}\right],
\end{aligned}
$$

Введенный здесь размерный нормировочный множитель $A$ будем выбирать, исходя из условий, при которых усредненная по периоду $T$ энергия электромагнитного поля в рассматриваемом конечном объеме $V$ фиксирована и равна $W_{0}$, а отнесенные к $A$ нормированные по энергии комплексные амплитуды, зависящие от координат, удовлетворяют следующему условию:

$$
\frac{1}{4} \int_{V} d \mathbf{x}\left[\mathbf{E}^{*}(\mathbf{x}) \mathbf{D}(\mathbf{x})+\mathbf{H}^{*}(\mathbf{x}) \mathbf{B}(\mathbf{x})+k . c .\right]=1,
$$

где $d \mathbf{x}=d x d y d z$ - элемент объема. В соответствии с этими требованием будем иметь

$$
\begin{aligned}
W_{0} & =\frac{1}{8 \pi} \frac{1}{T} \int_{0}^{T} d t \int_{V} d \mathbf{x}[\mathbf{E}(\mathbf{x}, t) \mathbf{D}(\mathbf{x}, t)+\mathbf{H}(\mathbf{x}, t) \mathbf{B}(\mathbf{x}, t)] \\
& =\frac{A^{2}}{8 \pi} \int_{V} d \mathbf{x}\left[\mathbf{E}^{*}(\mathbf{x}) \mathbf{D}(\mathbf{x})+\mathbf{H}^{*}(\mathbf{x}) \mathbf{B}(\mathbf{x})+k . c .\right]=\frac{A^{2}}{2 \pi} .
\end{aligned}
$$

Отсюда следует, что $A=\sqrt{2 \pi W_{0}}$, а равенство (3) можно рассматривать как условие нормировки энергии электромагнитной поля, выраженной через нормированные поля, на единицу.

Согласно (1), нормированные условием (3) комплексные амплитуды полей удовлетворяют следующим стационарным уравнениям Максвелла:

$$
\begin{gathered}
\nabla \times \mathbf{H}(\mathbf{x})=-i \frac{\omega}{c} \mathbf{D}(\mathbf{x}), \\
\nabla \times \mathbf{E}(\mathbf{x})=i \frac{\omega}{c} \mathbf{B}(\mathbf{x}) .
\end{gathered}
$$

В непоглощающей неоднородной анизотропной среде векторы индукции $\mathbf{D}(\mathbf{x})$ и $\mathbf{B}(\mathbf{x})$ связаны с напряженностями соответствующих полей $\mathbf{E}(\mathbf{x})$ и $\mathbf{H}(\mathbf{x})$ соотношениями

$$
\begin{aligned}
& D_{i}(\mathbf{x})=\sum_{j} \varepsilon_{i j}(\mathbf{x}) E_{j}(\mathbf{x}), \\
& B_{i}(\mathbf{x})=\sum_{j} \mu_{i j}(\mathbf{x}) H_{j}(\mathbf{x}) .
\end{aligned}
$$

Здесь $\varepsilon_{i j}(\mathbf{x})$ и $\mu_{i j}(\mathbf{x})$ зависящие от координат компоненты положительно определенных эрмитовых матриц тензоров диэлектрической и магнитной проницаемостей соответственно.
Покажем, что уравнения (4) совместно с уравнениями связи (5) являются одним из возможных представлений более общих операторных уравнений

$$
\begin{aligned}
i c(\hat{\mathbf{s}} \cdot \hat{\mathbf{k}})|H\rangle & =\omega \hat{\varepsilon}|E\rangle, \\
-i c(\hat{\mathbf{s}} \cdot \hat{\mathbf{k}})|E\rangle & =\omega \hat{\mu}|H\rangle,
\end{aligned}
$$

которые будем рассматривать как одночастичные квантово-механические уравнения для фотона в анизотропной среде. В этих уравнениях $\hat{\mathbf{s}}-$ оператор спина фотона; $\hat{\mathbf{k}}$ - оператор волнового вектора с точностью до постоянной Планка, совпадающий с оператором импульса $\hat{\mathbf{p}}=\hbar \hat{\mathbf{k}} ; \hat{\varepsilon}$ и $\hat{\mu}-$ соответственно операторы диэлектрической и магнитной проницаемостей. Все перечисленные операторы эрмитовы, т.е. $\hat{\mathbf{s}}^{+}=\hat{\mathbf{s}}, \hat{\mathbf{k}}^{+}=\hat{\mathbf{k}}$, $\hat{\varepsilon}^{+}=\hat{\varepsilon}, \hat{\mu}^{+}=\hat{\mu}$. В соответствии с общими принципами квантовой теории оператор $(\hat{\mathbf{s}} \cdot \hat{\mathbf{k}})$ следует интерпретировать как оператор спин-орбитального взаимодействия фотона. Напряженностям электрического и магнитного полей в этих уравнениях сопоставляются два вектора в комплексном гильбертовом пространстве $|E\rangle$ и $|H\rangle$, характеризующие стационарные состояния фотона в неоднородной анизотропной среде.

В качестве базиса представления уравнений (6) рассмотрим полный ортонормированный набор состояний фотона с определенной координатой $|\mathbf{x}\rangle$ и определенной линейной поляризацией вдоль вещественных ортов декартовой системы координат $\mathbf{e}_{i}=|i\rangle, \mathbf{e}_{i}=\langle i|(i=1,2,3$ или $x, y, z)$. Набор векторов $|\mathbf{x}, i\rangle=|\mathbf{x}\rangle \otimes|i\rangle$ в рассматриваемом гильбертовом пространстве образуют базис, удовлетворяющий соответственно следующим условиям ортонормированности и полноты:

$$
\begin{gathered}
\left\langle\mathbf{x}, i \mid \mathbf{x}^{\prime}, j\right\rangle=\delta\left(\mathbf{x}-\mathbf{x}^{\prime}\right) \delta_{i j}, \\
\sum_{i} \int_{V} d \mathbf{x}|\mathbf{x}, i\rangle\langle\mathbf{x}, i|=1 .
\end{gathered}
$$

Используя этот базис, представим векторы $|E\rangle$ и $|H\rangle$, а также сопряженные с ними векторы $\langle E|$ и $\langle H|$ в виде следующих разложений:

$$
\begin{aligned}
|E\rangle & =\sum_{i} \int_{V} d \mathbf{x}|\mathbf{x}, i\rangle\langle\mathbf{x}, i \mid E\rangle=\sum_{i} \int_{V} d \mathbf{x}|\mathbf{x}, i\rangle E_{i}(\mathbf{x}), \\
|H\rangle & =\sum_{i} \int_{V} d \mathbf{x}|\mathbf{x}, i\rangle\langle\mathbf{x}, i \mid H\rangle=\sum_{i} \int_{V} d \mathbf{x}|\mathbf{x}, i\rangle H_{i}(\mathbf{x}), \\
\langle E| & =\sum_{i} \int_{V} d \mathbf{x}\langle E \mid \mathbf{x}, i\rangle\langle\mathbf{x}, i|=\sum_{i} \int_{V} d \mathbf{x} E_{i}^{*}(\mathbf{x})\langle\mathbf{x}, i|, \\
\langle H| & =\sum_{i} \int_{V} d \mathbf{x}\langle H \mid \mathbf{x}, i\rangle\langle\mathbf{x}, i|=\sum_{i} \int_{V} d \mathbf{x} H_{i}^{*}(\mathbf{x})\langle\mathbf{x}, i| .
\end{aligned}
$$

Аналогичным образом определим в рассматриваемом базисе вид всех операторов, входящих в уравнения (6):

$$
\begin{gathered}
\left\langle\mathbf{x}, i|\hat{\mathbf{s}}| \mathbf{x}^{\prime}, j\right\rangle=\mathbf{s}_{i j} \delta\left(\mathbf{x}-\mathbf{x}^{\prime}\right), \\
\left\langle\mathbf{x}, i|\hat{\mathbf{k}}| \mathbf{x}^{\prime}, j\right\rangle=-i\left[\nabla_{\mathbf{x}} \delta\left(\mathbf{x}-\mathbf{x}^{\prime}\right)\right] \delta_{i j},
\end{gathered}
$$




$$
\begin{aligned}
\left\langle\mathbf{x}, i|\hat{\varepsilon}| \mathbf{x}^{\prime}, j\right\rangle & =\varepsilon_{i j}(\mathbf{x}) \delta\left(\mathbf{x}-\mathbf{x}^{\prime}\right), \\
\left\langle\mathbf{x}, i|\hat{\mu}| \mathbf{x}^{\prime}, j\right\rangle & =\mu_{i j}(\mathbf{x}) \delta\left(\mathbf{x}-\mathbf{x}^{\prime}\right) .
\end{aligned}
$$

Здесь $\mathbf{s}_{i j}$ - матричные элементы матриц спинового момента $s=1$ в декартовом базисе $|i\rangle[15]$ :

$$
s_{x}=\left(\begin{array}{ccc}
0 & 0 & 0 \\
0 & 0 & -i \\
0 & i & 0
\end{array}\right), s_{y}=\left(\begin{array}{ccc}
0 & 0 & i \\
0 & 0 & 0 \\
-i & 0 & 0
\end{array}\right), s_{z}=\left(\begin{array}{ccc}
0 & -i & 0 \\
i & 0 & 0 \\
0 & 0 & 0
\end{array}\right)
$$

Из полученных соотношений (7)-(10) следует, что

$$
\begin{aligned}
& \sum_{j} \int_{V} d \mathbf{x}^{\prime}\left\langle\mathbf{x}, i|(\hat{\mathbf{s}} \cdot \hat{\mathbf{k}})| \mathbf{x}^{\prime}, j\right\rangle\left\langle\mathbf{x}^{\prime}, j \mid H\right\rangle=[\nabla \times \mathbf{H}(\mathbf{x})]_{i}, \\
& \sum_{j} \int_{V} d \mathbf{x}^{\prime}\left\langle\mathbf{x}, i|(\hat{\mathbf{s}} \cdot \hat{\mathbf{k}})| \mathbf{x}^{\prime}, j\right\rangle\left\langle\mathbf{x}^{\prime}, j \mid E\right\rangle=[\nabla \times \mathbf{E}(\mathbf{x})]_{i}, \\
& \sum_{j} \int_{V} d \mathbf{x}^{\prime}\left\langle\mathbf{x}, i|\hat{\varepsilon}| \mathbf{x}^{\prime}, j\right\rangle\left\langle\mathbf{x}^{\prime}, j \mid E\right\rangle=\sum_{j} \varepsilon_{i j}(\mathbf{x}) E_{j}(\mathbf{x}), \\
& \sum_{j} \int_{V} d \mathbf{x}^{\prime}\left\langle\mathbf{x}, i|\hat{\mu}| \mathbf{x}^{\prime}, j\right\rangle\left\langle\mathbf{x}^{\prime}, j \mid H\right\rangle=\sum_{j} \mu_{i j}(\mathbf{x}) H_{j}(\mathbf{x}) .
\end{aligned}
$$

Тем самым доказывается эквивалентность операторных уравнений (6) в базисе $|\mathbf{x}, i\rangle$ и максвелловских уравнений (4).

Представление оператора спина $\hat{\mathbf{s}}$ в декартовом базисе $|i\rangle$ (формула (10)) не является единственно возможным. В гиротропных диэлектрических или магнитных средах с осью гирации, направленной вдоль оси $z$, более удобно использовать так называемый сферический базис $|m\rangle$ $(m=+1,0,-1)$, определяющий для электромагнитной волны, волновой вектор которой $\mathbf{k} \| z$, две ортогональные круговые поляризации: правую $(m=+1)$ и левую $(m=-1)$. Оба базиса декартов $|i\rangle$ и сферический $|m\rangle$ связаны следующим унитарным преобразованием [15]:

$$
\begin{gathered}
|i\rangle=\sum_{m}|m\rangle\langle m \mid i\rangle=\sum_{m}|m\rangle U_{m i}, \\
|m\rangle=\sum_{i}|i\rangle\langle i \mid m\rangle=\sum_{i}|i\rangle U_{i m}^{+},
\end{gathered}
$$

где

$$
\mathbf{U}=\left(\begin{array}{ccc}
-1 / \sqrt{2} & i & 0 \\
0 & 0 & 1 \\
1 / \sqrt{2} & i & 0
\end{array}\right)
$$

- унитарная матрица, удовлетворяющая условиям: $\mathbf{U} \cdot \mathbf{U}^{+}=\mathbf{U}^{+} \cdot \mathbf{U}=\mathbf{I}(\mathbf{I}-$ единичная матрица $)$. В частности, согласно (11) и (12):

$$
\begin{array}{r}
|+1\rangle=-\frac{|x\rangle+i|y\rangle}{\sqrt{2}}, \quad\langle+1|=-\frac{\langle x|-i\langle y|}{\sqrt{2}}, \\
|0\rangle=|z\rangle, \quad\langle 0|=\langle z|, \\
|-1\rangle=\frac{|x\rangle-i|y\rangle}{\sqrt{2}}, \quad\langle-1|=\frac{\langle x|+i\langle y|}{\sqrt{2}} .
\end{array}
$$

Множество векторов $|\mathbf{x}, m\rangle=|\mathbf{x}\rangle \otimes|m\rangle$ также образуют полный ортонормированный базис

$$
\begin{gathered}
\left\langle\mathbf{x}, m \mid \mathbf{x}^{\prime}, m^{\prime}\right\rangle=\delta\left(\mathbf{x}-\mathbf{x}^{\prime}\right) \delta_{m m^{\prime}}, \\
\sum_{m} \int_{V} d \mathbf{x}|\mathbf{x}, m\rangle\langle\mathbf{x}, m|=1 .
\end{gathered}
$$

Операторы в этом представлении имеют вид, аналогичный (9):

$$
\begin{gathered}
\left\langle\mathbf{x}, m|\hat{\mathbf{s}}| \mathbf{x}^{\prime}, m^{\prime}\right\rangle=\mathbf{s}_{m m^{\prime}}^{\prime} \delta\left(\mathbf{x}-\mathbf{x}^{\prime}\right) \\
\left\langle\mathbf{x}, m|\hat{\mathbf{k}}| \mathbf{x}^{\prime}, m^{\prime}\right\rangle=-i\left[\nabla_{\mathbf{x}} \delta\left(\mathbf{x}-\mathbf{x}^{\prime}\right)\right] \delta_{m m^{\prime}} \\
\left\langle\mathbf{x}, m|\hat{\varepsilon}| \mathbf{x}^{\prime}, m^{\prime}\right\rangle=\varepsilon_{m m^{\prime}}^{\prime}(\mathbf{x}) \delta\left(\mathbf{x}-\mathbf{x}^{\prime}\right) \\
\left\langle\mathbf{x}, m|\hat{\mu}| \mathbf{x}^{\prime}, m^{\prime}\right\rangle=\mu_{m m^{\prime}}^{\prime}(\mathbf{x}) \delta\left(\mathbf{x}-\mathbf{x}^{\prime}\right)
\end{gathered}
$$

где матрицы в сферическом базисе $\mathbf{s}^{\prime}, \boldsymbol{\varepsilon}^{\prime}(\mathbf{x}), \boldsymbol{\mu}^{\prime}(\mathbf{x})$ связаны с соответствующими матрицами в декартовом базисе $\mathbf{s}, \boldsymbol{\varepsilon}(\mathbf{x}), \boldsymbol{\mu}(\mathbf{x})$ унитарным преобразованием вида $\mathbf{c}^{\prime}=\mathbf{U} \cdot \mathbf{c} \cdot \mathbf{U}^{+}$. При этом матрицы спинового момента в результате этого преобразования приобретают вид

$$
\begin{gathered}
s_{x}^{\prime}=\frac{1}{\sqrt{2}}\left(\begin{array}{ccc}
0 & 1 & 0 \\
1 & 0 & 1 \\
0 & 1 & 0
\end{array}\right), \quad s_{y}^{\prime}=\frac{1}{\sqrt{2}}\left(\begin{array}{ccc}
0 & -i & 0 \\
i & 0 & -i \\
0 & i & 0
\end{array}\right), \\
s_{z}^{\prime}=\left(\begin{array}{ccc}
1 & 0 & 0 \\
0 & 0 & 0 \\
0 & 0 & -1
\end{array}\right),
\end{gathered}
$$

а матрицы $\boldsymbol{\varepsilon}(\mathbf{x})$ и $\boldsymbol{\mu}(\mathbf{x})$ для сред, у которых $\varepsilon_{11}(\mathbf{x})=\varepsilon_{22}(\mathbf{x})$ и $\mu_{11}(\mathbf{x})=\mu_{22}(\mathbf{x})$, а ось гирации направлена вдоль оси $z$, становятся диагональными.

\section{2. Переход к задаче определения собственных векторов и собственных чисел эрмитовых операторов}

Наличие в правых частях уравнений (6) операторов $\hat{\varepsilon}$ и $\hat{\mu}$ приводит к необходимости решения обобщенной задачи нахождения собственных чисел $\omega_{l}$ и собственных векторов $\left|E_{l}\right\rangle$ и $\left|H_{l}\right\rangle$. Однако посредством преобразований, она может быть сведена к обычной задаче определения собственных чисел и собственных векторов эрмитова оператора. С этой целью введем новые векторы состояний

$$
\begin{aligned}
|\tilde{E}\rangle & =\hat{\varepsilon}^{1 / 2}|E\rangle, \\
|\tilde{H}\rangle & =\hat{\mu}^{1 / 2}|H\rangle,
\end{aligned}
$$

а также неэрмитов оператор частоты $\hat{\Omega}$, который определим как

$$
\begin{gathered}
\hat{\Omega}=i c \hat{\varepsilon}^{-1 / 2}(\hat{\mathbf{s}} \cdot \hat{\mathbf{k}}) \hat{\mu}^{-1 / 2}, \\
\hat{\Omega}^{+}=-i c \hat{\mu}^{-1 / 2}(\hat{\mathbf{s}} \cdot \hat{\mathbf{k}}) \hat{\varepsilon}^{-1 / 2} .
\end{gathered}
$$


Новые векторы состояний, согласно (6), (13) и (14), удовлетворяют следующей системе операторных уравнений:

$$
\begin{aligned}
\hat{\Omega}|\tilde{H}\rangle & =\omega|\tilde{E}\rangle, \\
\hat{\Omega}^{+}|\tilde{E}\rangle & =\omega|\tilde{H}\rangle,
\end{aligned}
$$

а также эквивалентным ей уравнениям:

$$
\begin{gathered}
\hat{\Omega}^{+} \hat{\Omega}|\tilde{H}\rangle=\omega^{2}|\tilde{H}\rangle, \\
\hat{\Omega} \hat{\Omega}^{+}|\tilde{E}\rangle=\omega^{2}|\tilde{E}\rangle .
\end{gathered}
$$

Каждое из уравнений (16) является уравнением для определения собственных чисел (квадратов частот) $\omega_{l}^{2}$ и соответствующих им собственных векторов $\left|\tilde{H}_{l}\right\rangle$ и $\left|\tilde{E}_{l}\right\rangle$ эрмитовых операторов $\hat{\Omega}^{+} \hat{\Omega}$ и $\hat{\Omega} \hat{\Omega}^{+}$. Однако состояния $\left|\tilde{H}_{l}\right\rangle$ и $\left|\tilde{E}_{l}\right\rangle$ не независимы, а связанны между собой соотношениями (15). Как собственные векторы эрмитовых операторов они по отдельности ортогональны друг другу, если $l \neq l^{\prime}$, и могут быть нормированы на единицу

$$
\begin{aligned}
& \left\langle\tilde{E}_{l} \mid \tilde{E}_{l^{\prime}}\right\rangle=\delta_{l l^{\prime}}, \\
& \left\langle\tilde{H}_{l} \mid \tilde{H}_{l^{\prime}}\right\rangle=\delta_{l l^{\prime}} .
\end{aligned}
$$

Каждому собственному состоянию фотона $\left|\tilde{H}_{l}\right\rangle$ и $\left|\tilde{E}_{l}\right\rangle$ и в выбранном базисе будут соответствовать собственные нормированные по энергии электромагнитные моды. Так, например, в базисе $|\mathbf{x}, i\rangle$ им соответствуют моды $\tilde{H}_{i, l}(\mathbf{x})=\left\langle\mathbf{x}, i \mid \tilde{H}_{l}\right\rangle$ и $\tilde{E}_{i, l}(\mathbf{x})=\left\langle\mathbf{x}, i \mid \tilde{E}_{l}\right\rangle$.

Покажем, что условия нормировки (17) эквивалентны условию нормировки усредненной по периоду энергии электромагнитного поля, запасенной в $l$-й собственной моде, на единицу. Действительно, согласно (3), усредненная по периоду плотность энергии $l$-й моды $w_{l}(\mathbf{x})$, выраженная через нормированные поля, определяется соотношением

$$
w_{l}(\mathbf{x})=\frac{1}{4}\left[\mathbf{E}_{l}^{*}(\mathbf{x}) \cdot \mathbf{D}_{l}(\mathbf{x})+\mathbf{H}_{l}^{*}(\mathbf{x}) \cdot \mathbf{B}_{l}(\mathbf{x})+k . c .\right] .
$$

Откуда следует, что

$$
\begin{aligned}
W_{l} & =\int_{V} d \mathbf{x} w_{l}(\mathbf{x}) \\
& =\frac{1}{2} \sum_{i, j} \int_{V} d \mathbf{x}\left[E_{i l}^{*}(\mathbf{x}) \varepsilon_{i j}(\mathbf{x}) E_{j l}(\mathbf{x})+H_{i l}^{*}(\mathbf{x}) \mu_{i j}(\mathbf{x}) H_{j l}(\mathbf{x})\right] \\
& =\frac{1}{2}\left[\left\langle E_{l}|\hat{\varepsilon}| E_{l}\right\rangle+\left\langle H_{l}|\hat{\mu}| H_{l}\right\rangle\right]=\frac{1}{2}\left[\left\langle\tilde{E}_{l} \mid \tilde{E}_{l}\right\rangle+\left\langle\tilde{H}_{l} \mid \tilde{H}_{l}\right\rangle\right]=1 .
\end{aligned}
$$

При выводе этих соотношений мы воспользовались формулами (5), (8), (9), (13) и (17). Аналогичным образом для каждой $l$-й собственной моды определим усредненные по периоду плотность импульса электромагнитного поля $\mathbf{S}_{l}(\mathbf{x})$, которая пропорциональна вектору Пойнтинга, и плотность спинового момента $\mathbf{M}_{l}(\mathbf{x})$ :

$$
\begin{aligned}
\mathbf{S}_{l}(\mathbf{x}) & =\frac{1}{2}\left[\mathbf{E}_{l}^{*}(\mathbf{x}) \times \mathbf{H}_{l}(\mathbf{x})-\mathbf{H}_{l}^{*}(\mathbf{x}) \times \mathbf{E}_{l}(\mathbf{x})\right], \\
\mathbf{M}_{l}(\mathbf{x}) & =\frac{-i}{2}\left[\tilde{\mathbf{E}}_{l}^{*}(\mathbf{x}) \times \tilde{\mathbf{E}}_{l}(\mathbf{x})+\tilde{\mathbf{H}}_{l}^{*}(\mathbf{x}) \times \tilde{\mathbf{H}}_{l}(\mathbf{x})\right] .
\end{aligned}
$$

$\mathrm{B}$ соответствии с этим полный импульс $\mathbf{S}_{l}$ и полный спиновый момент $\mathbf{M}_{l}$ нормированной по энергии моды будут определяться следующими выражениями:

$$
\begin{gathered}
\mathbf{S}_{l}=\int_{V} d \mathbf{x} \mathbf{S}_{l}(\mathbf{x})=\frac{i}{2}\left(\left\langle E_{l}|\hat{\mathbf{s}}| H_{l}\right\rangle-\left\langle H_{l}|\hat{\mathbf{s}}| E_{l}\right\rangle\right), \\
\mathbf{M}_{l}=\int_{V} d \mathbf{x} \mathbf{S}_{l}(\mathbf{x})=\frac{1}{2}\left(\left\langle\tilde{E}_{l}|\hat{\mathbf{s}}| \tilde{E}_{l}\right\rangle+\left\langle\tilde{H}_{l}|\hat{\mathbf{s}}| \tilde{H}_{l}\right\rangle\right) .
\end{gathered}
$$

Как следует из этих формул, обе величины $\mathbf{S}_{l}$ и $\mathbf{M}_{l}$ являются безразмерными.

\section{3. Собственные моды анизотропного 3D ФK}

Рассмотрим общий случай анизотропного $3 D$ ФК, прямая и обратная решетки которого определяются тремя, связанными друг с другом, основными (примитивными) векторами трансляции $\mathbf{a}_{1,2,3}$ и $\mathbf{b}_{1,2,3}$ соответственно. Произвольные векторы трансляции прямой $\mathbf{a}$ и $\mathbf{b}$ обратной решеток определим как

$$
\begin{gathered}
\mathbf{a}=n_{1} \mathbf{a}_{1}+n_{2} \mathbf{a}_{2}+n_{3} \mathbf{a}_{3}, \\
\mathbf{b}=m_{1} \mathbf{b}_{1}+m_{2} \mathbf{b}_{2}+m_{3} \mathbf{b}_{3},
\end{gathered}
$$

где $n_{i}, m_{i}=0, \pm 1, \pm 2 \ldots(i=1,2,3)$. Согласно свойствам ФК, все операторы в уравнениях (15) и (16) должны обладать трансляционной симметрией, в частности, операторы диэлектрической и магнитной проницаемостей в базисе $|\mathbf{x}, i\rangle$ удовлетворять соотношениям

$$
\begin{aligned}
\varepsilon_{i j}(\mathbf{x}+\mathbf{a}) & =\varepsilon_{i j}(\mathbf{x}), \\
\mu_{i j}(\mathbf{x}+\mathbf{a}) & =\mu_{i j}(\mathbf{x}) .
\end{aligned}
$$

В трансляционно-инвариантной системе в качестве базиса представления уравнений (15) и (16) удобно использовать состояния фотона с определенным волновым вектором, который запишем в виде суммы вектора обратной решетки $\mathbf{b}$ и вектора $\mathbf{k}$, пробегающего для ограниченного кристалла дискретный ряд значений в зоне Бриллюэна ФК, и определенной, например, линейной поляризацией $|\mathbf{b}+\mathbf{k}, i\rangle$. Этот базис связан с базисом $|\mathbf{x}, i\rangle$ следующим унитарным преобразованием (преобразованием Фурье):

$$
|\mathbf{b}+\mathbf{k}, i\rangle=\sum_{j} \int_{V} d \mathbf{x}|\mathbf{x}, j\rangle\langle\mathbf{x}, j \mid \mathbf{b}+\mathbf{k}, i\rangle,
$$

где

$$
\langle\mathbf{x}, j \mid \mathbf{b}+\mathbf{k}, i\rangle=\frac{1}{\sqrt{V}} e^{i(\mathbf{b}+\mathbf{k}) \mathbf{x}} \delta_{j i},
$$

$V=N \Omega_{0}-$ объем $Ф К, N-$ число элементарных ячеек в $\Phi К, \Omega_{0}$ - объем элементарной ячейки. Базисные орты $|\mathbf{b}+\mathbf{k}, i\rangle$ удовлетворяют соответственно следующим условиям ортонормированности и полноты:

$$
\left\langle\mathbf{b}+\mathbf{k}, i \mid \mathbf{b}^{\prime}+\mathbf{k}^{\prime}, j\right\rangle=\delta_{\mathbf{b b}^{\prime}} \delta_{\mathbf{k k}^{\prime}} \delta_{i j},
$$




$$
\sum_{\mathbf{b}, \mathbf{k}, i}|\mathbf{b}+\mathbf{k}, i\rangle\langle\mathbf{b}+\mathbf{k}, i|=1
$$

Используя соотношения (20)-(23), определим вид всех интересующих нас операторов в этом базисе

$$
\begin{gathered}
\left\langle\mathbf{b}+\mathbf{k}, i|\hat{\mathbf{s}}| \mathbf{b}^{\prime}+\mathbf{k}^{\prime}, j\right\rangle=\mathbf{s}_{i j} \delta_{\mathbf{b b}^{\prime}} \delta_{\mathbf{k} \mathbf{k}^{\prime}} \\
\left\langle\mathbf{b}+\mathbf{k}, i|\hat{\mathbf{k}}| \mathbf{b}^{\prime}+\mathbf{k}^{\prime}, j\right\rangle=(\mathbf{b}+\mathbf{k}) \delta_{\mathbf{b b}^{\prime}} \delta_{i j} \delta_{\mathbf{k k}^{\prime}} \\
\left\langle\mathbf{b}+\mathbf{k}, i|\hat{\varepsilon}| \mathbf{b}^{\prime}+\mathbf{k}^{\prime}, j\right\rangle=\varepsilon_{i j}\left(\mathbf{b}-\mathbf{b}^{\prime}\right) \delta_{\mathbf{k k}^{\prime}} \\
\left\langle\mathbf{b}+\mathbf{k}, i|\hat{\mu}| \mathbf{b}^{\prime}+\mathbf{k}^{\prime}, j\right\rangle=\mu_{i j}\left(\mathbf{b}-\mathbf{b}^{\prime}\right) \delta_{\mathbf{k k}^{\prime}}
\end{gathered}
$$

Здесь $\mathbf{s}_{i j}$ определяются формулой (10), а

$$
\begin{aligned}
& \varepsilon_{i j}\left(\mathbf{b}-\mathbf{b}^{\prime}\right)=\frac{1}{\Omega_{0}} \int_{\Omega_{0}} d \mathbf{x} \varepsilon_{i j}(\mathbf{x}) e^{-i\left(\mathbf{b}-\mathbf{b}^{\prime}\right) \mathbf{x}}, \\
& \mu_{i j}\left(\mathbf{b}-\mathbf{b}^{\prime}\right)=\frac{1}{\Omega_{0}} \int_{\Omega_{0}} d \mathbf{x} \mu_{i j}(\mathbf{x}) e^{-i\left(\mathbf{b}-\mathbf{b}^{\prime}\right) \mathbf{x}}
\end{aligned}
$$

- фурье-образы компонент диэлектрической и магнитной проницаемостей соответственно.

Как следует из (24), наличие трансляционной симметрии у ФК приводит к тому, что все операторы в рассматриваемом базисе диагональны по индексам $\mathbf{k}$ и $\mathbf{k}^{\prime}$. В peзультате соответствующие этим операторам матрицы представления имеют блочно-диагональную структуру. Поэтому они сами и любые их произведения могут быть выражены через матрицы меньшей размерности, параметрически зависящие от волнового вектора $\mathbf{k}$. Так, например

$$
\left\langle\mathbf{b}+\mathbf{k}, i|\hat{\Omega}| \mathbf{b}^{\prime}+\mathbf{k}^{\prime}, j\right\rangle=\Omega_{\mathbf{b} i, \mathbf{b}^{\prime} j}(\mathbf{k}) \delta_{\mathbf{k k}^{\prime}},
$$

где $\Omega_{\mathbf{b} i, \mathbf{b}^{\prime} j}(\mathbf{k})$ - матричные элементы следующей матрицы (см. формулу (14)):

$$
\boldsymbol{\Omega}(\mathbf{k})=i c \boldsymbol{\varepsilon}^{-1 / 2}(\mathbf{s} \cdot \mathbf{K}(\mathbf{k})) \cdot \boldsymbol{\mu}^{-1 / 2} .
$$

Здесь и далее в этом разделе $\mathbf{s}, \mathbf{K}(\mathbf{k}), \boldsymbol{\varepsilon}$ и $\boldsymbol{\mu}$ матрицы, элементы которых, согласно (24), определены соответственно следующим образом:

$$
\begin{gathered}
\mathbf{s}_{\mathbf{b} i, \mathbf{b}^{\prime} j}=\mathbf{s}_{i j} \delta_{\mathbf{b b}^{\prime}}, \\
\mathbf{K}_{\mathbf{b} i, \mathbf{b}^{\prime} j}(\mathbf{k})=(\mathbf{b}+\mathbf{k}) \delta_{\mathbf{b b}^{\prime}} \delta_{i j}, \\
\varepsilon_{\mathbf{b} i, \mathbf{b}^{\prime} j}=\varepsilon_{i j}\left(\mathbf{b}-\mathbf{b}^{\prime}\right), \\
\mu_{\mathbf{b} i, \mathbf{b}^{\prime} j}=\varepsilon_{i j}\left(\mathbf{b}-\mathbf{b}^{\prime}\right) .
\end{gathered}
$$

Согласно вышеизложенному, собственные векторы $\left|\tilde{H}_{l \mathbf{k}^{\prime}}\right\rangle$ и $\left|\tilde{E}_{l \mathbf{k}^{\prime}}\right\rangle$, являющиеся решениями уравнений (15) и (16) и описывающие собственные $l \mathbf{k}^{\prime}$-е блоховские состояния фотона, в базисе $|\mathbf{b}+\mathbf{k}, i\rangle$ также имеют вид блочно-диагональных матриц:

$$
\begin{aligned}
\left\langle\mathbf{b}+\mathbf{k}, i \mid \tilde{H}_{l \mathbf{k}^{\prime}}\right\rangle & =\tilde{H}_{\mathbf{b} i, l}(\mathbf{k}) \delta_{\mathbf{k} \mathbf{k}^{\prime}}, \\
\left\langle\mathbf{b}+\mathbf{k}, i \mid \tilde{E}_{l \mathbf{k}^{\prime}}\right\rangle & =\tilde{E}_{\mathbf{b} i, l}(\mathbf{k}) \delta_{\mathbf{k k}^{\prime}} .
\end{aligned}
$$

Следовательно, в этом базисе они будут характеризоваться параметрически зависящими от волнового вектора $\mathbf{k}$ собственными столбцами $\tilde{\mathbf{H}}_{l}(\mathbf{k})$ и $\tilde{\mathbf{E}}_{l}(\mathbf{k})$, компоненты которых соответственно равны $\tilde{H}_{\mathbf{b} i, l}(\mathbf{k})$ и $\tilde{E}_{\mathbf{b} i, l}(\mathbf{k})$. В результате операторные уравнения (15) и (16) могут быть представлены соответственно в виде следующих параметрически зависящих от вектора $\mathbf{k}$ матричных уравнений:

$$
\begin{aligned}
\boldsymbol{\Omega}(\mathbf{k}) \cdot \tilde{\mathbf{H}}_{l}(\mathbf{k}) & =\omega_{l}(\mathbf{k}) \tilde{\mathbf{E}}_{l}(\mathbf{k}), \\
\mathbf{\Omega}^{+}(\mathbf{k}) \cdot \tilde{\mathbf{E}}_{l}(\mathbf{k}) & =\omega_{l}(\mathbf{k}) \tilde{\mathbf{H}}_{l}(\mathbf{k}) .
\end{aligned}
$$

и

$$
\begin{aligned}
\boldsymbol{\Omega}^{+}(\mathbf{k}) \cdot \boldsymbol{\Omega}(\mathbf{k}) \cdot \tilde{\mathbf{H}}_{l}(\mathbf{k}) & =\omega_{l}^{2}(\mathbf{k}) \tilde{\mathbf{H}}_{l}(\mathbf{k}), \\
\mathbf{\Omega}(\mathbf{k}) \cdot \boldsymbol{\Omega}^{+}(\mathbf{k}) \cdot \tilde{\mathbf{E}}_{l}(\mathbf{k}) & =\omega_{l}^{2}(\mathbf{k}) \tilde{\mathbf{E}}_{l}(\mathbf{k}) .
\end{aligned}
$$

Таким образом, задача определения собственных нормированных по энергии блоховских мод ФК сводится к поиску собственных чисел $\omega_{l}^{2}(\mathbf{k})$ и соответствующих им собственных столбцов $\tilde{\mathbf{H}}_{l}(\mathbf{k}), \tilde{\mathbf{E}}_{l}(\mathbf{k})$ матричных уравнений (28) и (29). Для этого, как указывалось выше, из четырех уравнений (28) и (29) достаточно рассмотреть только два, например, следующие:

$$
\begin{gathered}
\boldsymbol{\Omega}^{+}(\mathbf{k}) \cdot \boldsymbol{\Omega}(\mathbf{k}) \cdot \tilde{\mathbf{H}}_{l}(\mathbf{k})=\omega_{l}^{2}(\mathbf{k}) \tilde{\mathbf{H}}_{l}(\mathbf{k}), \\
\tilde{\mathbf{E}}_{l}(\mathbf{k})=\frac{1}{\omega_{l}(\mathbf{k})} \boldsymbol{\Omega}(\mathbf{k}) \cdot \tilde{\mathbf{H}}_{l}(\mathbf{k}) .
\end{gathered}
$$

При этом продольные моды, у которых $\omega_{l}(\mathbf{k})=0$, должны быть исключены из рассмотрения. Необходимую для определения матрицы $\boldsymbol{\Omega}(\mathbf{k})$ процедуру извлечения корня квадратного из эрмитовых матриц $\boldsymbol{\varepsilon}$ и $\boldsymbol{\mu}$ с элементами, определенными в (26), можно осуществить посредством предварительного перехода к базису, в котором эти матрицы диагональны, а после извлечения корня возвратиться к исходному базису. Собственные столбцы $\tilde{\mathbf{H}}_{l}(\mathbf{k})$ и $\tilde{\mathbf{E}}_{l}(\mathbf{k})$ удовлетворяют следующим условиям нормировки:

$$
\begin{array}{r}
\tilde{\mathbf{H}}_{l}^{+}(\mathbf{k}) \cdot \tilde{\mathbf{H}}_{l^{\prime}}(\mathbf{k})=\delta_{l l^{\prime}}, \\
\tilde{\mathbf{E}}_{l}^{+}(\mathbf{k}) \cdot \tilde{\mathbf{E}}_{l^{\prime}}(\mathbf{k})=\delta_{l l^{\prime}},
\end{array}
$$

которые непосредственно следуют из условий ортонормированности собственных блоховских состояний фотона $\left\langle\tilde{H}_{l \mathbf{k}} \mid \tilde{H}_{l^{\prime} \mathbf{k}^{\prime}}\right\rangle=\left\langle\tilde{E}_{l \mathbf{k}} \mid \tilde{E}_{l^{\prime} \mathbf{k}^{\prime}}\right\rangle=\delta_{l l^{\prime}} \delta_{\mathbf{k k}^{\prime}}$ и соотношений (27):

$$
\begin{aligned}
\left\langle\tilde{H}_{l \mathbf{k}} \mid \tilde{H}_{l^{\prime} \mathbf{k}^{\prime}}\right\rangle & =\sum_{\mathbf{b}, \mathbf{k}^{\prime \prime}, i}\left\langle\tilde{H}_{l \mathbf{k}} \mid \mathbf{b}+\mathbf{k}^{\prime \prime}, i\right\rangle\left\langle\mathbf{b}+\mathbf{k}^{\prime \prime}, i \mid \tilde{H}_{l^{\prime} \mathbf{k}^{\prime}}\right\rangle \\
& =\delta_{\mathbf{k} \mathbf{k}^{\prime}} \sum_{\mathbf{b}, i} \tilde{H}_{l, \mathbf{b} i}^{+}(\mathbf{k}) \tilde{H}_{\mathbf{b} i, l^{\prime}}(\mathbf{k}) \\
& =\delta_{\mathbf{k} \mathbf{k}^{\prime}} \tilde{\mathbf{H}}_{l}^{+}(\mathbf{k}) \cdot \tilde{\mathbf{H}}_{l^{\prime}}(\mathbf{k})=\delta_{\mathbf{k k}^{\prime}} \delta_{l l^{\prime}} .
\end{aligned}
$$

Аналогичным образом доказывается и второе условие в (31).

Пространственное распределение проекций магнитного и электрического полей в $l \mathbf{k}$-й нормированной 
по энергии блоховской моде $H_{i, l \mathbf{k}}(\mathbf{x})=\left\langle\mathbf{x}, i \mid H_{l \mathbf{k}}\right\rangle$ и $E_{i, l \mathbf{k}}(\mathbf{x})=\left\langle\mathbf{x}, i \mid E_{l \mathbf{k}}\right\rangle$ можно определить с помощью обратного преобразования Фурье:

$$
\begin{aligned}
& H_{i, l \mathbf{k}}(\mathbf{x})=\frac{1}{\sqrt{V}} \sum_{\mathbf{b}} e^{i(\mathbf{b}+\mathbf{k}) \mathbf{x}} H_{\mathbf{b} i, l}(\mathbf{k})=\frac{1}{\sqrt{N}} e^{i \mathbf{k x}} H_{i, l}(\mathbf{x} ; \mathbf{k}), \\
& E_{i, l \mathbf{k}}(\mathbf{x})=\frac{1}{\sqrt{V}} \sum_{\mathbf{b}} e^{i(\mathbf{b}+\mathbf{k}) \mathbf{x}} E_{\mathbf{b} i, l}(\mathbf{k})=\frac{1}{\sqrt{N}} e^{i \mathbf{k x}} E_{i, l}(\mathbf{x} ; \mathbf{k}) .
\end{aligned}
$$

Здесь

$$
\begin{gathered}
H_{i, l}(\mathbf{x} ; \mathbf{k})=\frac{1}{\sqrt{\Omega_{0}}} \sum_{\mathbf{b}} e^{i \mathbf{b x}} H_{\mathbf{b} i, l}(\mathbf{k}), \\
E_{i, l}(\mathbf{x} ; \mathbf{k})=\frac{1}{\sqrt{\Omega_{0}}} \sum_{\mathbf{b}} e^{i \mathbf{b x}} E_{\mathbf{b} i, l}(\mathbf{k})
\end{gathered}
$$

- периодические части блоховских функций (32), фурье-компоненты которых $H_{\mathbf{b} i, l}(\mathbf{k})$ и $E_{\mathbf{b} i, l}(\mathbf{k})$ образуют соответственно столбцы $\mathbf{H}_{l}(\mathbf{k})$ и $\mathbf{E}_{l}(\mathbf{k})$, которые, согласно (13), связаны с собственными столбцами матричных уравнений $(30) \tilde{\mathbf{H}}_{l}(\mathbf{k})$ и $\tilde{\mathbf{E}}_{l}(\mathbf{k})$ соотношениями

$$
\begin{aligned}
\mathbf{H}_{l}(\mathbf{k}) & =\boldsymbol{\mu}^{-1 / 2} \cdot \tilde{\mathbf{H}}_{l}(\mathbf{k}), \\
\mathbf{E}_{l}(\mathbf{k}) & =\boldsymbol{\varepsilon}^{-1 / 2} \cdot \tilde{\mathbf{H}}_{l}(\mathbf{k})
\end{aligned}
$$

где матрицы $\boldsymbol{\mu}$ и $\boldsymbol{\varepsilon}$ определены в (26).

При вычислении полного импульса и полного спинового момента $l \mathbf{k}$-й нормированной по энергии блоховской моды достаточно воспользоваться формулами (18) и (19). Соответственно будем иметь

$$
\begin{array}{r}
\mathbf{S}_{l}(\mathbf{k})=\frac{i}{2}\left[\mathbf{E}_{l}^{+}(\mathbf{k}) \cdot \mathbf{s} \cdot \mathbf{H}_{l}(\mathbf{k})-\mathbf{H}_{l}^{+}(\mathbf{k}) \cdot \mathbf{s} \cdot \mathbf{E}_{l}(\mathbf{k})\right], \\
\mathbf{M}_{l}(\mathbf{k})=\frac{1}{2}\left[\tilde{\mathbf{E}}_{l}^{+}(\mathbf{k}) \cdot \mathbf{s} \cdot \tilde{\mathbf{E}}_{l}(\mathbf{k})+\tilde{\mathbf{H}}_{l}^{+}(\mathbf{k}) \cdot \mathbf{s} \cdot \tilde{\mathbf{H}}_{l}(\mathbf{k})\right],
\end{array}
$$

где мы воспользовались матричным представлением оператора спина (формула (26)).

Покажем, что полный импульс $l \mathbf{k}$-й нормированной по энергии моды $\mathbf{S}_{l}(\mathbf{k})$ совпадает с ее групповой скоростью $\mathbf{v}_{g, l}(\mathbf{k})=\partial \omega_{l}(\mathbf{k}) / \partial \mathbf{k}$, отнесенной к скорости света в вакууме. С этой целью в уравнениях (28) осуществим бесконечно малую вариацию волнового вектора $\delta \mathbf{k}[16]$. Будем иметь

$$
\begin{aligned}
\delta \boldsymbol{\Omega}(\mathbf{k}) \cdot \tilde{\mathbf{H}}_{l}(\mathbf{k}) & +\boldsymbol{\Omega}(\mathbf{k}) \cdot \delta \tilde{\mathbf{H}}_{l}(\mathbf{k})=\delta \omega_{l}(\mathbf{k}) \tilde{\mathbf{E}}_{l}(\mathbf{k}) \\
& +\omega_{l}(\mathbf{k}) \delta \tilde{\mathbf{E}}_{l}(\mathbf{k}), \\
\delta \mathbf{\Omega}^{+}(\mathbf{k}) \cdot \tilde{\mathbf{E}}_{l}(\mathbf{k}) & +\boldsymbol{\Omega}^{+}(\mathbf{k}) \cdot \delta \tilde{\mathbf{E}}_{l}(\mathbf{k})=\delta \omega_{l}(\mathbf{k}) \tilde{\mathbf{H}}_{l}(\mathbf{k}) \\
& +\omega_{l}(\mathbf{k}) \delta \tilde{\mathbf{H}}_{l}(\mathbf{k}),
\end{aligned}
$$

Далее умножим слева первое из этих уравнений на $\tilde{\mathbf{E}}_{l}^{+}(\mathbf{k})$, а второе на $\tilde{\mathbf{H}}_{l}^{+}(\mathbf{k})$ и воспользуемся уравнениями для эрмитово сопряженных столбцов

$$
\begin{gathered}
\tilde{\mathbf{H}}_{l}^{+}(\mathbf{k}) \cdot \boldsymbol{\Omega}^{+}(\mathbf{k})=\omega_{l}(\mathbf{k}) \tilde{\mathbf{E}}_{l}^{+}(\mathbf{k}), \\
\tilde{\mathbf{E}}_{l}^{+}(\mathbf{k}) \cdot \boldsymbol{\Omega}(\mathbf{k})=\omega_{l}(\mathbf{k}) \tilde{\mathbf{H}}_{l}^{+}(\mathbf{k}),
\end{gathered}
$$

которые получаются посредством эрмитова сопряжения уравнений (28). Складывая левые и правые части полученных уравнений после сокращений подобных членов и учета условий нормировки (31), получим

$\tilde{\mathbf{E}}_{l}^{+}(\mathbf{k}) \cdot \delta \boldsymbol{\Omega}(\mathbf{k}) \cdot \tilde{\mathbf{H}}_{l}(\mathbf{k})+\tilde{\mathbf{H}}_{l}^{+}(\mathbf{k}) \cdot \delta \boldsymbol{\Omega}^{+}(\mathbf{k}) \cdot \tilde{\mathbf{E}}_{l}(\mathbf{k})=2 \delta \omega_{l}(\mathbf{k})$.

Согласно (25) и (26):

$$
\begin{gathered}
\delta \boldsymbol{\Omega}(\mathbf{k})=i c \boldsymbol{\varepsilon}^{-1 / 2} \cdot(\mathbf{s} \cdot \delta \mathbf{k}) \cdot \boldsymbol{\mu}^{-1 / 2}, \\
\delta \boldsymbol{\Omega}^{+}(\mathbf{k})=-i c \boldsymbol{\mu}^{-1 / 2} \cdot(\mathbf{s} \cdot \delta \mathbf{k}) \cdot \boldsymbol{\varepsilon}^{-\mathbf{1 / 2}},
\end{gathered}
$$

Подстановка (37) в (36) с учетом (34) и (35) приводит к искомому результату

$$
\mathbf{S}_{l}(\mathbf{k})=\mathbf{v}_{g, l}(\mathbf{k}) / c .
$$

\section{4. Дисперсия собственных мод анизотропных и гиротропных $1 D \Phi \mathrm{K}$ (численный расчет)}

Развитая выше теория была использована для расчета зонной структуры и пространственного распределения собственных мод $1 D$ ФК на основе анизотропных и гиротропных диэлектрических и магнитных сред. Рассматривался наиболее общий случай среды, с периодически изменяющимися в направлении $z$ компонентами тензоров диэлектрической $\varepsilon_{i k}(z)$ и магнитной $\mu_{i k}(z)$ проницаемостей. С этой целью были введены две периодические с периодом решетки ФК $a_{0}$ ступенчатые функции

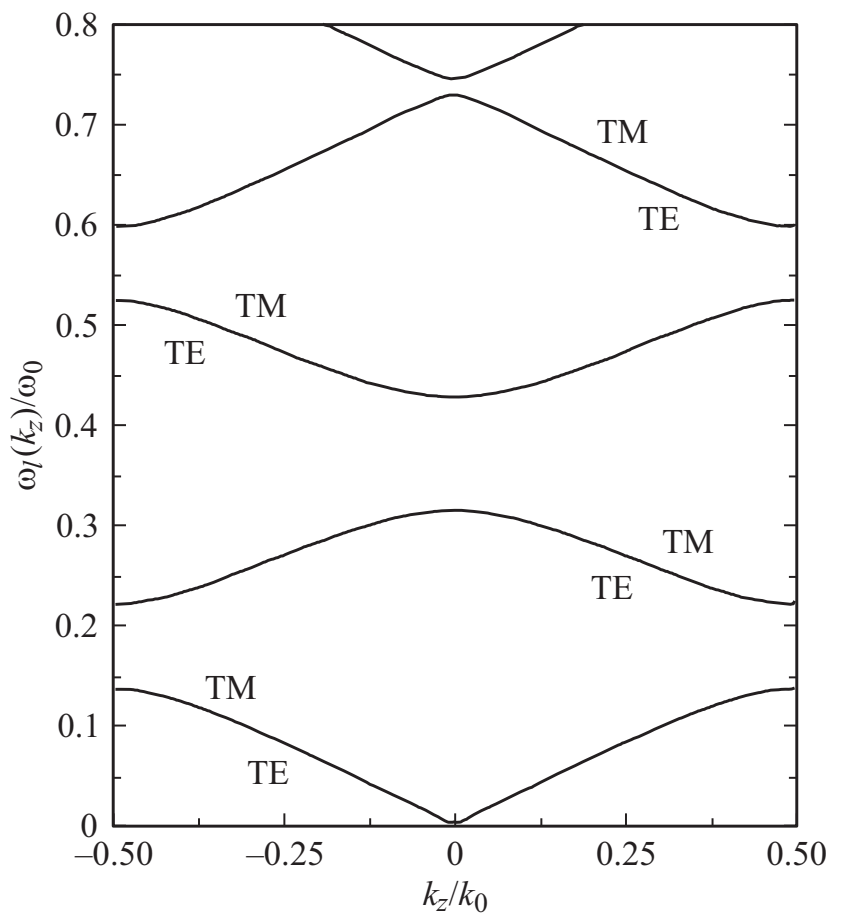

Рис. 1. Зонная структура изотропного $1 D$ ФК. Параметры ФК: $\Delta \varepsilon_{i k}^{1}=15 \delta_{i k}, \Delta \varepsilon_{i k}^{2}=\delta_{i k}, \Delta \mu_{i k}^{1,2}=0 ; \omega_{0}=c k_{0}, k_{0}=2 \pi / a_{0}$. 

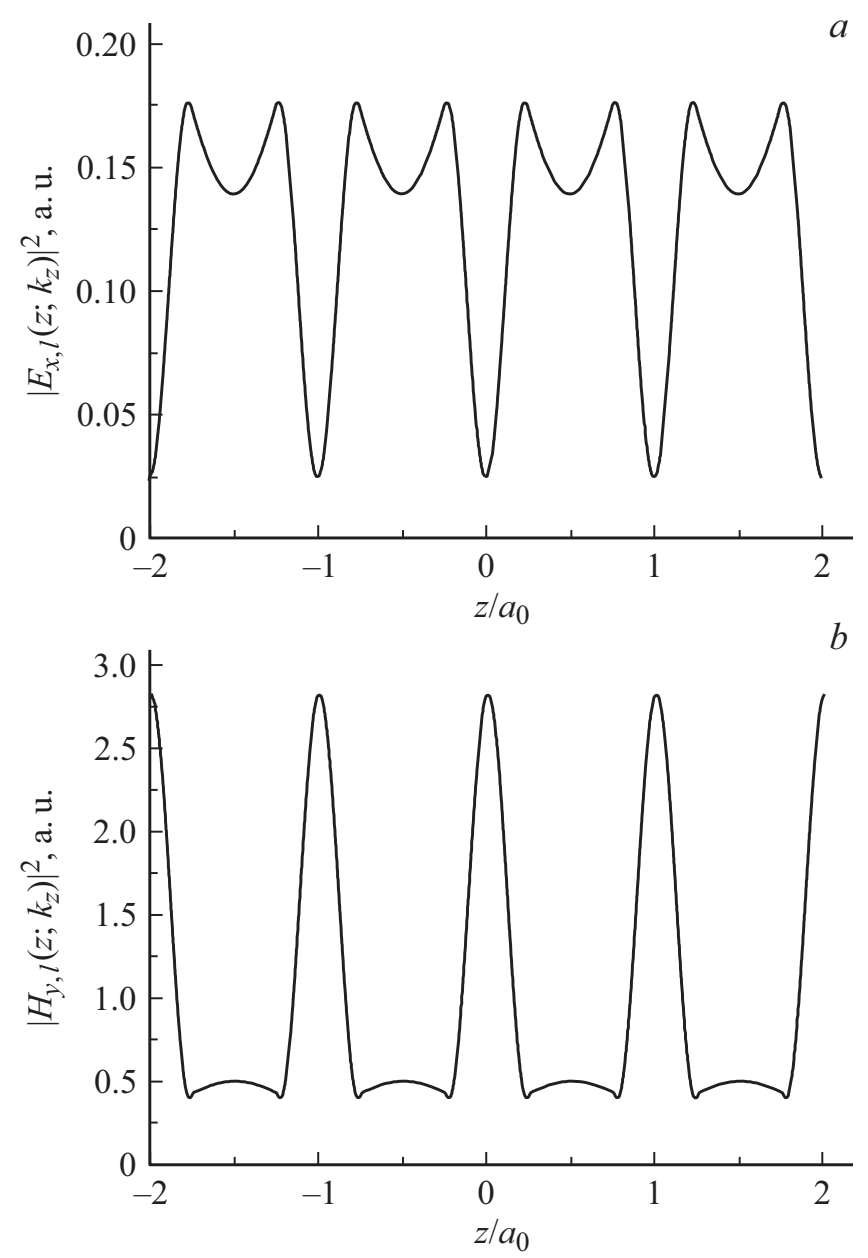

Рис. 2. Пространственное распределение электрического $(a)$ и магнитного $(b)$ полей в изотропном $1 D$ ФК. ТМ-мода, $l=3$, $k_{z}=0.25 k_{0}$. Параметры ФК приведены в подписи к рис. 1.

$f_{1}(z)$ и $f_{2}(z)=1-f_{1}(z)$, осуществляющие послойную пространственную модуляцию как диэлектрической, так и магнитной проницаемостей. Первая из этих функций в пределах элементарной ячейки ФК, центрированной в точке $z=0$, удовлетворяла следующим условиям: $f_{1}(z)=1$ в области $|z| \leq a_{0} / 4$ и $f_{1}(z)=0$ в остальной ее части. Координатная зависимость компонент тензоров определялась следующим образом:

$$
\begin{aligned}
& \varepsilon_{i k}(z)=\delta_{i k}+\Delta \varepsilon_{i k}^{1} f_{1}(z)+\Delta \varepsilon_{i k}^{2} f_{2}(z), \\
& \mu_{i k}(z)=\delta_{i k}+\Delta \mu_{i k}^{1} f_{1}(z)+\Delta \mu_{i k}^{2} f_{2}(z) .
\end{aligned}
$$

Здесь $\Delta \varepsilon_{i k}^{1,2}$ и $\Delta \mu_{i k}^{1,2}-$ постоянные, характеризующие изменения компонент тензоров относительно вакуума соответственно в слоях 1 и 2. Произвольный вектор обратной решетки, направленный воль оси $z$, определялся как $b_{m}=\left(2 \pi / a_{0}\right) m$, где $m=-N_{b} \ldots 0 \ldots N_{b}$ $\left(N_{b}=80\right)$. Таким образом, матрица $\boldsymbol{\Omega}(\mathbf{k})$ имела размерность $483 \times 483$. Во всех расчетах рассматривались электромагнитные волны, у которых $k_{x}=k_{y}=0$.

В качестве примера на рис. 1 представлены результаты расчета зонной структуры изотропного $1 D$ ФК, параметры которого указаны в подписи к рисунку. Как и следовало ожидать, собственные моды этого кристалла, соответствующие ТМ- и ТЕ-поляризациям вырождены. Пространственное распределение электромагнитного поля в этом кристалле рассчитывалось по формуле (33). Соответствующие результаты для квадрата модуля амплитуд поля ТМ-моды приведены на рис. 2.

На рис. 3 представлены результаты расчета зонной структуры ФК с анизотропной диэлектрической проницаемостью в обоих слоях, но различной ориентацией в них осей анизотропии. Как видно из рисунка, наряду с расщеплением собственных мод, обусловлен-

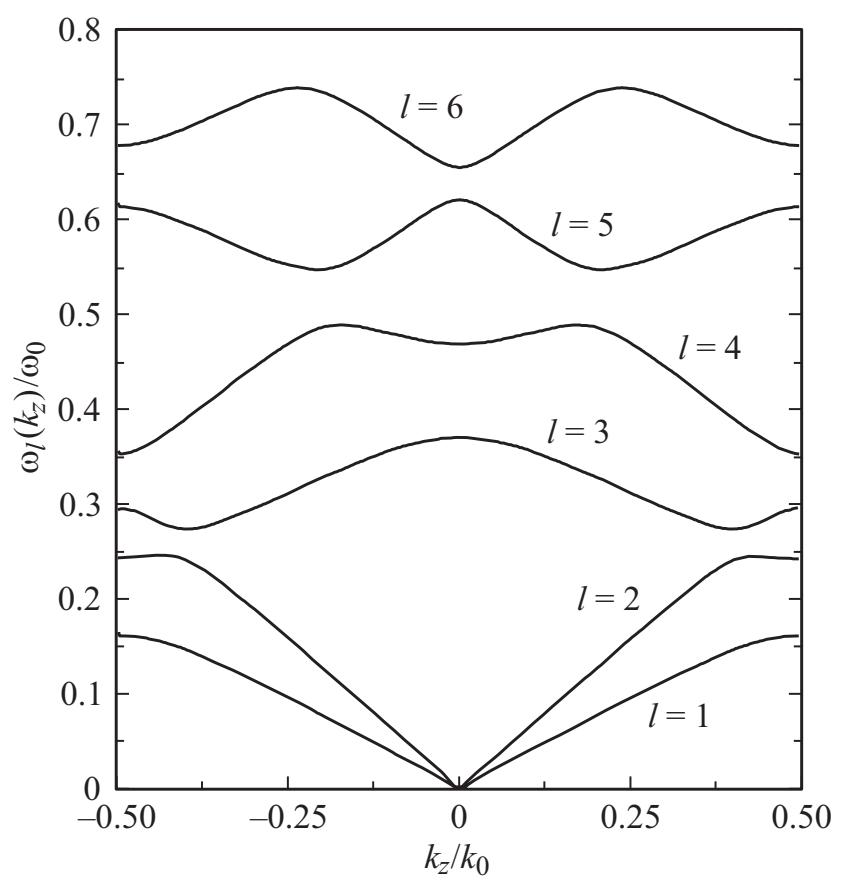

Рис. 3. Зонная структура анизотропного $1 D$ ФК. Параметры $\quad$ ФК: $\quad \Delta \varepsilon_{11}^{1}=10, \quad \Delta \varepsilon_{22}^{1}=\Delta \varepsilon_{33}^{1}=2, \quad \Delta \varepsilon_{i k}^{1}=0$ $(i \neq k), \Delta \varepsilon_{11}^{2}=\Delta \varepsilon_{22}^{2}=\Delta \varepsilon_{33}^{2}=\Delta \varepsilon_{12}^{2}=\Delta \varepsilon_{21}^{2}=1, \Delta \varepsilon_{i 3}^{2}=\Delta \varepsilon_{3 i}^{2}=0$ $(i=1,2), \Delta \mu_{i k}^{1,2}=0$.

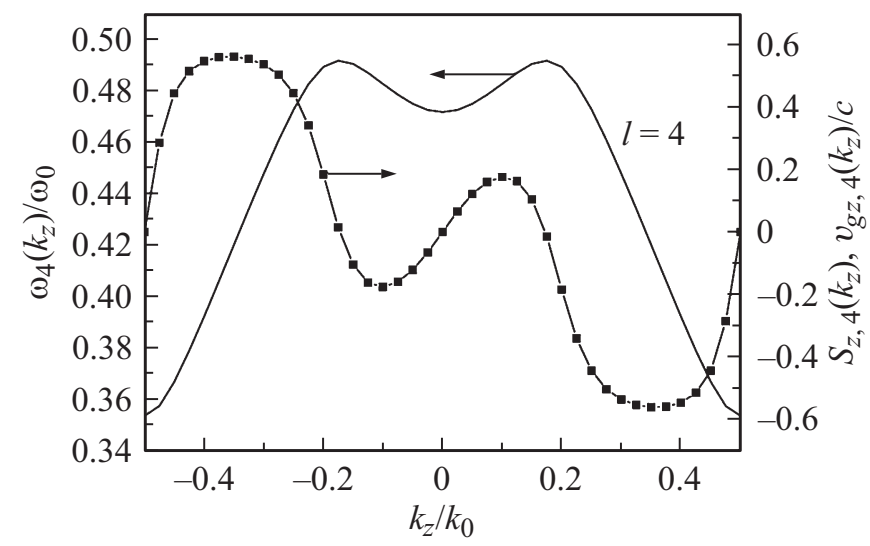

Рис. 4. Дисперсия частоты, полного импульса и групповой скорости моды $l=4$ анизотропного $1 D$ ФК. Параметры ФК приведены в подписи к рис. 3. Точками показана дисперсия групповой скорости. 


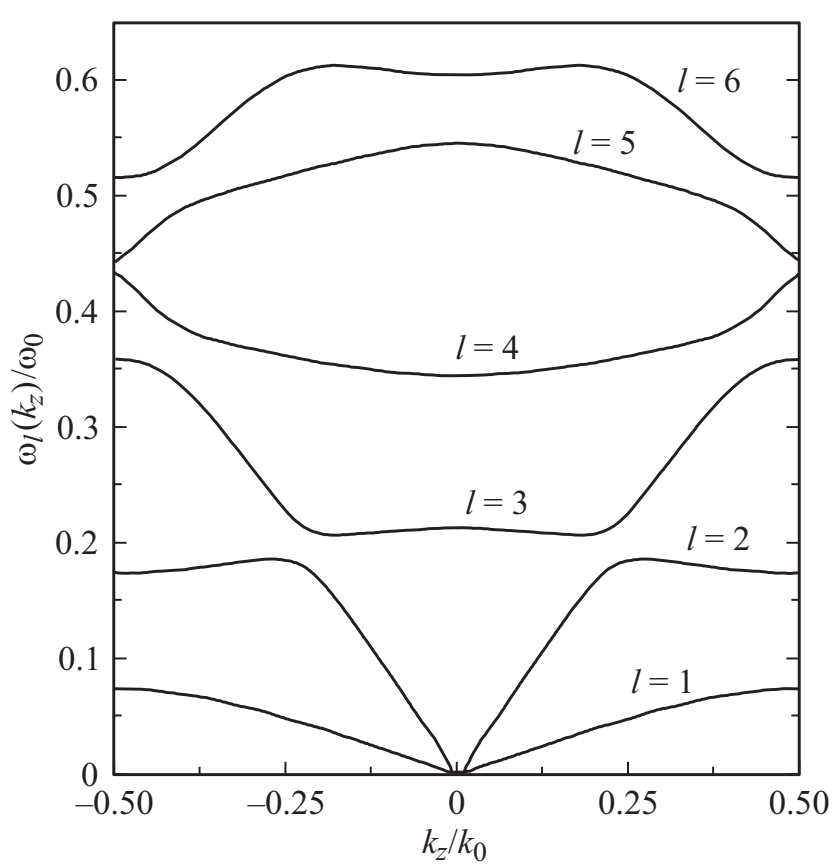

Рис. 5. Зонная структура гиротропного $1 D$ ФК. Параметры ФК: $\quad \Delta \varepsilon_{i k}^{1}=0, \quad \Delta \varepsilon_{11}^{2}=\Delta \varepsilon_{22}^{2}=\Delta \varepsilon_{33}^{2}=\Delta \varepsilon_{12}^{2}=\Delta \varepsilon_{21}^{2}=1$, $\Delta \varepsilon_{i 3}^{2}=\Delta \varepsilon_{3 i}^{2}=0 \quad(i=1,2), \quad \Delta \mu_{11}^{1}=\Delta \mu_{22}^{1}=\Delta \mu_{33}^{1}=15$, $\Delta \mu_{12}^{1}=-\Delta \mu_{21}^{1}=15 i, \Delta \mu_{i 3}^{1}=\Delta \mu_{3 i}^{1}=0(i=1,2), \Delta \mu_{i k}^{2}=0$.

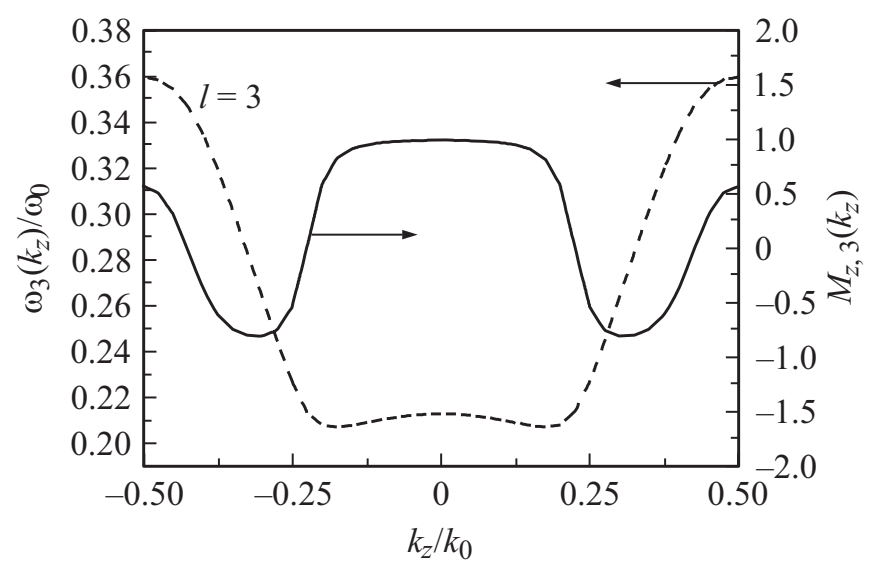

Рис. 6. Дисперсия частоты и полного момента моды $l=3$ гиротропного $1 D$ ФК. Параметры ФК приведены в подписи к рис. 5. Дисперсия частоты показана штриховой линией.

ным анизотропией диэлектрических проницаемостей, в этом случае дополнительно наблюдаются эффекты антипересечения дисперсионных ветвей. Рассчитанная по формуле (35) зависимость проекции полного импульса электромагнитного поля на ось $z S_{z, l}\left(k_{z}\right)$ от волнового вектора $k_{z}$ моды с номером $l=4$ представлена на рис. 4. Для сравнения на этом же рисунке приведены закон дисперсии этой моды $\omega_{4}\left(k_{z}\right)$ и рассчитанная с его помощью дисперсия ее групповой скорости $v_{g z, 4}\left(k_{z}\right)$. Как видно, дисперсионные зависимости полного импульса и групповой скорости, отнесенной к скорости света в вакууме, совпадают, что находится в полном согласии с представленными выше теоретическими результатами (формула (38)).

Дисперсия собственных мод ФК при наличии магнитной гиротропии одного из его слоев и диэлектрической анизотропии другого слоя, ось анизотропии которого не совпадает с осью гирации, приведена на рис. 5. Здесь, как и в случае анизотропного ФК (рис. 3), наблюдается снятие вырождения собственных мод и антипересечение дисперсионных ветвей. В качестве примера на рис. 6 представлен результат расчета зависимости проекции полного спинового момента на ось $z M_{z, l}\left(k_{z}\right)$ моды с номером $l=3$ (формула (35)). Здесь же для сравнения приведен ее закон дисперсии $\omega_{3}\left(k_{z}\right)$. Из рисунка следует, что спиновый момент переносимый этой модой зависит от $k_{z}$ и в наших безразмерных единицах изменяется в пределах зоны Бриллюэна от -1 , что соответствует волнам с левой круговой поляризацией, до +1 , что характерно для волн с правой круговой поляризацией.

\section{Заключение}

В работе продемонстрирована эквивалентность стационарных уравнений Максвелла для комплексных амплитуд электромагнитного поля и одночастичных квантовомеханических операторных уравнений для фотона. Переход к операторным уравнениям существенно упростил и максимально формализовал задачу определения зонной структуры и собственных мод ФК. Кроме того, данный подход позволил с единых позиций анализировать ФК на основе периодических сред с произвольной анизотропией и гиротропией их диэлектрических и магнитных свойств. Эффективность предлагаемого метода продемонстрирована на примере анализа зонной структуры и характеристик собственных мод анизотропных и гиротропных $1 D \Phi К$.

Автор признателен чл.-корр. РАН Ивченко Е.Л. и Глазову М.М. за интерес к настоящей работе и обсуждение ее результатов. Работа выполнена в рамках государственного задания Министерства образования и науки РФ (проектная часть 16.1750.2017/4.6).

\section{Список литературы}

[1] Yablonovitch E. // Phys. Rev. Lett. 1987. Vol. 58. P. 20592062.

[2] John S. // Phys. Rev. Lett. 1987. Vol. 58. P. 2486-2489.

[3] Sukhoivanov I.A., Guryev I.V. Photonic Crystals. Springer, 2009. $242 \mathrm{p}$.

[4] Sakoda K. Optical Properties of Photonic Crystals. Springer, 2005. 253 p.

[5] Спицын А.С., Глинский ГФ. // ЖТФ. 2008. Т. 78. Вып. 5. C. 71-77. [Spitsyn A.S., Glinskii G.F. // Techn. Phys. 2008. Vol. 53. N 5. P. 602-608. DOI: 10.1134/S1063784208050125]

[6] Спицын А.С., Глинский Г.Ф. // ФТП. 2008. Т. 42. Вып. 10. C. 1261-1267. [Spitsyn A.S., Glinskii G.F. // Semiconductors. 2008. Vol. 42. N 10. P. 1237-1243. DOI: $10.1134 / \mathrm{S} 1063782608100175]$ 
[7] Li Z-Y., Wang J., Gu D-Y. // Phys. Rev. B. 1998. Vol. 58. P. 3721-3729.

[8] Belotelov V.I., Zvezdin A.K. // J. Opt. Soc. Am. B. 2005. Vol. 22. N 1. P. 286-292.

[9] Alagappan G., Sun X.W., Shum P., Yu M.B., Doan M.T. // J. Opt. Soc. Am. B. 2006. Vol. 23. N 1. P. 159-167.

[10] Alagappan G., Sun X.W., Sun H.D. // Phys. Rev. B. 2008. Vol. 77. P. 195117. DOI: 10.1103/PhysRevB.77.195117

[11] Khromova I.A., Melnikov L.A. // Opt. Commun. 2008. Vol. 281. P. 5458-5466. DOI: $10.1016 /$ j.optcom.2008.07.059

[12] Panah M.B., Abrishamian M.S., Mirtaheri S.A. // J. Opt. 2011. Vol. 13. P. 015103. DOI: 10.1088/2040-8978/13/1/015103

[13] Ignatov A.I., Merzlikin A.M., Levy M., Vinogradov A.P. // Materials. 2012. Vol. 5. P. 1055-1083.

DOI: $10.3390 / \mathrm{ma} 5061055$

[14] Zhang H.-F., Liu S.-B., Kong X.-K. // Phys. Plasmas. 2013. Vol. 20. P. 092105.

[15] Глинский Г.Ф. Методы теории групп в квантовой механике. СПб.: Изд-во СПбГЭТУ „ЛЭТИ“, 2012. 200 с. (http://www.twirpx.com/file/1014645/)

[16] Глинский Г.Ф. // Изв. вузов. Радиофизика. 1980. Т. 23. № 1. C. $90-95$. 